\title{
MULTI-CRITERIA PROGRAMMING METHODS AND PRODUCTION PLAN OPTIMIZATION PROBLEM SOLVING IN METAL INDUSTRY
}

Tunjo Perić1
Željko Mandić
Original scientific paper

DOI:10.21554/hrr.091707

Faculty of Economics and Business Zagreb, University of Zagreb, Croatia

ALFE-MI d.o.o. Živinice, Bosnia and Herzegovina

Received: 18.08 .2017

Accepted: 06.09.2017

\begin{abstract}
This paper presents the production plan optimization in the metal industry considered as a multi-criteria programming problem. We first provided the definition of the multi-criteria programming problem and classification of the multicriteria programming methods. Then we applied two multi-criteria programming methods (the STEM method and the PROMETHEE method) in solving a problem of multi-criteria optimization production plan in a company from the metal industry. The obtained results indicate a high efficiency of the applied methods in solving the problem.
\end{abstract}

\section{INTRODUCTION}

Multi criteria programming is a complex process of determining non-dominated(efficient) solutions from a set of feasible solutions and determining the preferred solution from a set of non-dominated solutions. The basic stages of multi-criteria programming are:

1.Defining system goals and determining ways to achieve these goals

2. Mathematical description of the system and definition of the method of evaluation of the criteria functions

3. Application of existing multi criteria programming methods

4. Making a final decision

5. If the final solution is not accepted, retrieving new information and repeating the process from stage 2 by redefining the task (Opricović, 1986).

When solving problems with multi criteria functions, which are at the same time conflicting, decision maker wishes to achieve more than by using only one criterion in selecting of the course of action, satisfying the conditions dictated by the environment, processes, and resources (Perić, 2008).

The multi criteria programming is one of the most stud- ied areas of operational research. Numerous methods of multi-criteria programming have been developed, however, we still have a small number of real-world applications of multi-criteria programming. The multi criteria programming is a process with active participation of the decision maker who should recognize the best solution or give some information of his preferences connected to criteria functions. Our goal is to show that we can apply multicriteria programming methods to solve the problems of production planning in the metal industry in a situation where the decision maker is unable to identify the best non-dominated solution and provide additional information about preferences of criteria functions.

This paper investigates the possibility and efficiency of application oftwomulti-criteria programming methods (STEM and PROMETHEE) in order to optimize the production plan in a firm from metal industry: the STEM method is used to form the set of non-dominated solutions, while the PROMETHEE method is used to choose the preferred solution from the set of non-dominated solutions.

\section{${ }^{1}$ Correspodence to:}

Tunjo Perić, Faculty of Economics and Business Zagreb, University of Zagreb, Croatia

Trg J. F. Kenedija, 10000, Zagreb, Croatia

E-mail: tperic@efzg.hr 
The rest of the paper is organized as follows. In the section 2 the methodology of multi criteria programming used in the section 3 is presented. In section 3 an application of the two multi criteria programming methods is given. At the end the conclusion and references are given.

\section{METHODOLOGY OF MULTI-CRITERIA PROGRAMMING}

Solving the problem of maximizing/minimizing multiple criteria functions on a set of feasible solutions requires the formation of a multi-criteria programming model.

Multicriteria programming model defines a programming model with two or more criteria functions on a set of feasible solutions. The mathematical form of this model can be presented in the following way:

$$
\max _{\mathbf{x} \in \mathbf{S}} \mathbf{f}(\mathbf{x})=\left(f_{1}(\mathbf{x}), f_{2}(\mathbf{x}), \ldots, f_{K}(\mathbf{x})\right)^{T}(K \geq 2)
$$

where

$\mathbf{S}=\left\{\mathbf{x}: \mathbf{g}(\mathbf{x})=\left(g_{1}(\mathbf{x}), g_{2}(\mathbf{x}), \ldots, g_{m}(\mathbf{x})\right)^{T} \leq \mathbf{0}\right\}, \mathbf{x}=\left(x_{1}, x_{2}, \ldots, x_{n}\right)^{T}$, and $\mathrm{o}$ is a $\mathrm{m}$-dimensional null-vector.

From the relation (1) we can see that in the multi criteria programming model there exist a set of $\mathrm{K}$ criteria functions which should be maximized (if we have the functions that need to be minimized in the model, it is enough to multiply these functions by -1 , mconstraints and $n$ variables. If the multi criteria model contains only linear functions $f(x)$ and $\mathrm{g}(\mathrm{x})$ than we have multi criteria linear programming model. However, if some of these functions are nonlinear, it is a non-linear multi criteria programming model (Perić, 2008).

The decision maker has a key role in solving the multi criteria programming problems. Solving a multi criteria programming problem requires the cooperation of the decision maker and an analyst. The analyst is a person or a computer program responsible for the mathematical aspect of the solution process. "The analyst generates information for the decision maker to consider and the solution is selected according to the preferences of the decision maker" (Miettinen, 1998).

\section{The main multi criteria programming terms}

In the literature dealing with multi criteria programming we encounter the following terms:

\section{Definition 1: The feasible set $S$}

The feasible set $\mathbf{S}$ is the set of $\mathbf{x}$ vectors, $\mathbf{x} \in \mathbf{R}^{n}$. which satisfy the constraints $\mathbf{g}(\mathbf{x}) \leq \mathbf{0}$

$\mathbf{g} \in \mathbf{R}^{m}$, i.e. $\mathbf{S}=\{\mathbf{x}: \mathbf{g}(\mathbf{x}) \leq \mathbf{0}\}$.

\section{Definition 2: The criteria set $\mathbf{F}$}

Each element of $\mathbf{S}$ is associated with the vector $\mathbf{f}(\mathbf{x})$, meaning that it is possible to map $\mathbf{S}$, $\mathbf{S} \subset \mathbf{R}^{n}$, to $\mathbf{F}, \mathbf{F} \subset \mathbf{R}^{K}$, in the criterion function space. $\mathbf{F}$ is a criteria set that can be defined as $\mathbf{F}=\{\mathbf{f}(\mathbf{x}): \mathbf{x} \in \mathbf{S}\}$.

\section{Definition 3: Optimal (marginal) solution}

Optimal (marginal) solution represents the maximum of each component of the vector $\mathbf{f}(\mathbf{x})$ on a set of feasible solutions $\mathrm{S}$, i.e.

$$
\max _{\mathbf{x} \in \mathbf{S}} f_{k}(\mathbf{x})=f_{k}\left(\mathbf{x}^{k^{*}}\right)=f_{k}^{*} .
$$

\section{Definition 4. The perfect solution}

The perfect solution of the multi criteria programming model is one that simultaneously gives the maximal value of each criteria function on the given set $\mathbf{S}$. So $\mathbf{x}^{*}$ is the perfect solution of the given model if and only if $\mathbf{x}^{*} \in \mathbf{S}$ and $\mathbf{f}\left(\mathbf{x}^{*}\right) \geq \mathbf{f}(\mathbf{x})$ for each $\mathbf{x} \in \mathbf{S}$. Since it is in the nature of the multi criteria programming models that have conflicting goals, they generally do not have the perfect solution, respectively it is unfeasible.

Definition 5. The ideal value of the vector function $\mathbf{f}(\mathbf{x})$ (ideal)

The vector $\mathbf{f}^{*}(\mathbf{x})=\left(f_{1}^{*}(\mathbf{x}), f_{2}^{*}(\mathbf{x}), \ldots, f_{K}^{*}(\mathbf{x})\right)$ whose $\mathrm{k}^{\text {th }}$ component is the extreme value of the function $f_{k}(\mathbf{x}), \mathrm{k}=1, \ldots, \mathrm{K}$, at the set $\mathrm{S}$, is called the ideal value of the vector function $\mathbf{f}(\mathbf{x})$.

Definition 6. Non-dominated and weakly nondominated solution

$\mathbf{f}^{\prime \prime} \in \mathbf{F}$ is non-dominated if and only if does not exist another $\mathbf{f} \in \mathbf{F}$ such that $\mathbf{f} \geq \mathbf{f}^{*}$ and $\mathbf{f} \neq \mathbf{f}^{*}$ (i.e. $f_{k} \geq f_{k}^{*}$ for all $k=1, \ldots, K$ and $f_{k} \neq f_{k}^{*}$

for some $\mathrm{k})$.

$\mathbf{f}^{*} \in \mathbf{F}$ is weakly non-dominated if and only if does not exist another $\mathbf{f} \in \mathbf{F}$ such that $\mathbf{f}>\mathbf{f}^{*}$ (i.e. $\left.f_{k}>f_{k}^{*}, k=1, \ldots, K\right)$.

Definition 7. Efficient and weakly efficient solution

$\mathbf{x}^{*} \in \mathbf{S}$ is efficient if and only if there does not exist another $\mathbf{x} \in \mathbf{S}$ such that $\mathbf{f}(\mathbf{x}) \geq \mathbf{f}\left(\mathbf{x}^{*}\right)$ and $\mathbf{f}(\mathbf{x}) \neq \mathbf{f}\left(\mathbf{x}^{*}\right)$. $\mathbf{x}^{*} \in \mathbf{S}$ is weaklyefficient if and only if there does not exist another $\mathbf{x} \in \mathbf{S}$ such that $\mathbf{f}(\mathbf{x})>\mathbf{f}^{*}=\mathbf{f}\left(\mathbf{x}^{*}\right)$.

\section{Definition 8. Preferred solution}

The preferred solution is a non-dominated solution chosen by the decision maker, by using of some other criteria, as final. 
As such, it lies in an acceptable area for the value of all the criteria functions of the given model. The preferred solution is known as the 'best compromise solution' (Perić, 2008).

\section{Multi-criteria programming methods}

To solve multi criteria programming models there are many different methods. The most of the existing methods involve active participation of the decision maker in the solving process. The obtained preferred solution depends on the information received from the decision maker. There are many classifications of multi criteria programming methods in the literature. The most important review of methods and their classifications are given in: Hwang and Masud (1979), Zionts (1980), Chankong and Himes (1983), Steuer (1985), Lai and Hwang (1996), Perić (2008).

All known classifications are essentially different because they are based on a different set of methods and different classification criteria.

According to the nature of variables in the system to be optimized all multi criteria programming methods can be divided into deterministic and stochastic ones. According to the quality of elementary activities, all multi criteria programming methods can be classified as methods with continuous variables and as those with discrete variables, and according to the criterion of linearity of criteria functions and constraints on linear and nonlinear multi-criteria programming methods (Perić, 2008).

According to the number of possible solutions, we can divide the deterministic multi criteria programming methods into: (1) methods for determining one or more non-dominated solutions and (2) methods for selecting a preferred solution from the final set of non-dominated solutions.

According to the criterion of the existence and character of the preferences of the decision-maker, we can classify the deterministic multi criteria programming methods with continuous variables for determining one or more non-dominated solutions into four groups:

1. Methods where there is no clearly expressed preference of the decision maker

2. Methods where there is a clearly expressed preference of the decision-maker

3. Interactive methods

4. Methods with a posteriori expressed preference of the decision maker (Perić, 2008).

Particularly interesting are interactive methods that we can combine with methods for selecting a preferred solution from a set of non-dominated solutions. Here, we will present the STEM method as an interactive method of forming a set of non-dominated solutions and PROMETHEE method as a method for selecting a preferred solution from a set of non-dominated solutions.

\section{The STEMMethod}

The STEM method was proposed by Benayoun, de Montgolfier, Tergny, and Larichev (1971). Itis an interactive method intented to solve multi-criteria linear programming models.

In the STEM method each iteration (cycle) contains two phases: (1) calculation phase and (2) decision phase. In the calculation phase in the cycle $p$ we should find a feasible solution which is the "closest" to the ideal solution $\mathrm{fk}^{*}(\mathrm{k}=1,2, \ldots, \mathrm{K})$ by solving the following linear programming model:

$$
\min _{(\mathbf{x}, \lambda) \in \mathbf{S}_{p}} \lambda,
$$

where

$$
\begin{aligned}
& \mathbf{S}_{p}=\left\{(\mathbf{x}, \lambda) \in \mathbf{R}^{n+1}: \mathbf{x} \in \mathbf{S} ; \lambda \geq\left|f_{k}^{*}-f_{k}(\mathbf{x})\right| \cdot \pi_{k}, \lambda \geq 0, k=1,2, \ldots, K\right\} \\
& \pi_{k}=\alpha_{k} / \sum_{k=1}^{K} \alpha_{k}, \alpha_{k}=\frac{f_{k}^{*}-f_{k}^{\min }}{f_{k}^{*}}\left[1 / \sqrt{\sum_{j=1}^{n}\left(c_{k j}\right)^{2}}\right], \text { if } f_{k}^{*} \\
& \alpha_{k}=\frac{f_{k}^{\min }-f_{k}^{*}}{f_{k}^{\min }}\left[1 / \sqrt{\sum_{j=1}^{n}\left(c_{k j}\right)^{2}}\right], \text { if } f_{k}^{*}<0 .
\end{aligned}
$$

The obtained compromise solution $\mathrm{x}_{\mathrm{p}}$ in the decision phase is presented to the DM who compare their criteria function $f_{k}$ with the ideal criteria function value $\mathrm{fk}^{*}$. If some of the criteria functions are satisfied, the decision maker must reduce the level of the satisfied criteria function in the amount to allow improving of the unsatisfactory criteria functions in the next step of the method. The decision maker gives $\Delta z_{k}$ as the amount of the acceptable alleviation.

For the next iterative cycle the feasible set is modified to

$\mathbf{S}_{p+1}=\left\{(\mathbf{x}, \lambda) \in \mathbf{S}_{p} ; f_{k}(\mathbf{x}) \geq f_{k}\left(\mathbf{x}^{p}\right)-\Delta f_{k} ; f_{i}(\mathbf{x}) \geq f_{i}\left(\mathbf{x}^{p}\right), k \neq i ; k, i=1, \ldots, K\right\}$. $\pi_{k}=0$ is determined and then starts the calculation phase of the cycle $p+1$. In the calculation phase the analyst can solve several linear programming problems with the feasible $\mathrm{S}_{\mathrm{p}}$ taking $\Delta f_{k}$ inputs so that $0<\Delta f_{k}^{1}<\Delta f_{k}^{2}<\ldots<\Delta f_{k}^{c}$. Alarge number of efficient solutions can be obtained in this way. 
The set of efficient solutions is presented to the DMs, out of which they can choose the preferred one (Hwang and Masud (1979).

\section{PROMETHEEMethod}

The PROMETHEE method (Preference Ranking Method for Enrichment Evaluations) was developed by Brans and Vincke (1984). This method is intended to rank the solutions within the final set of non-dominated solutions (alternatives) (Perić, 2008).

The algorithm of the PROMETHEE method consists of three segments:

\section{The first segment:}

Creating the preference of the decision-maker, whereby for each criterion, six possible inclusions based on the intensity of preference are observed. The inclusion of the criteria is based on introducing the preference function, which represents the decision-maker's preference for the solution (alternative) $\mathrm{x}^{1}$ in relation to the solution (alternative) $\mathrm{x}^{2}$. The preference func- tion is defined for each criteria function separately, and its value ranges from 0 to 1 . As this value is closer to 1 , the preference of the decision-maker is greater, and the lesser the value of this function, the greater the indifference it has.

Let $f(\cdot)$ denote a criteria function, and $\mathrm{x}^{1}$ and $\mathrm{x}^{2}$ are two solutions from the set $\mathbf{S}$. The associated preference function $\mathrm{P}\left(\mathrm{x}^{1}, \mathrm{x}^{2}\right)$ of $\mathrm{x}^{1}$ in relation to $\mathrm{x}^{2}$ is defined as: $P\left(\mathbf{x}^{1}, \mathbf{x}^{2}\right)=\left\{\begin{array}{cl}0 & \text { if } f\left(\mathbf{x}^{1}\right) \leq f\left(\mathbf{x}^{2}\right) \\ P\left(f\left(\mathbf{x}^{1}\right), f\left(\mathbf{x}^{2}\right)\right)=P\left(f\left(\mathbf{x}^{1}\right)-f\left(\mathbf{x}^{2}\right)\right) & \text { if } f\left(\mathbf{x}^{1}\right)>f\left(\mathbf{x}^{2}\right) .\end{array}\right.$ Studies have shown that six types of functions, presented in Table 1, cover the most practical cases for which the maximum of two parameters have to be defined by the decision maker, which is simply since each parameter has a real economic significance. In order to have a better overview of the indifference area, we can observe the function $H(\mathrm{~d}),\left(\mathrm{d}=\mathrm{f}\left(\mathrm{x}^{1}\right)\right.$ $\left.f\left(x^{2}\right)\right)$ that is directly related to the preference function $P$ as follows:

$$
H(d)= \begin{cases}P\left(\mathbf{x}^{1}, \mathbf{x}^{2}\right) & d \geq 0 \\ P\left(\mathbf{x}^{2}, \mathbf{x}^{1}\right) & d \leq 0\end{cases}
$$

Table 1 Types of generalised criteria $(P(d)$ : Preference function)

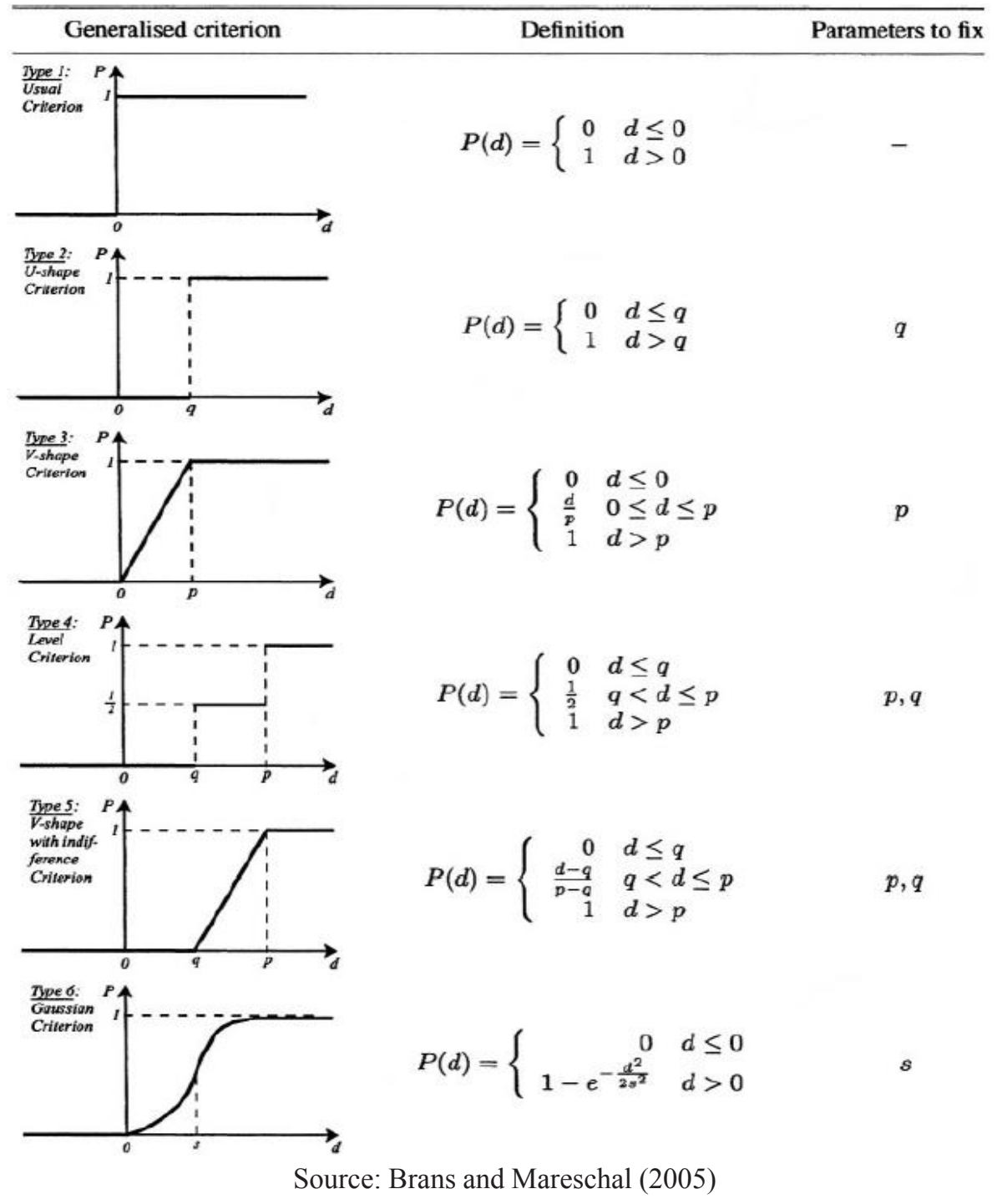


The decision maker defines 0,1 or 2 parameters: $q$ is a threshold of indifference;

$p$ is threshold of strict preference;

$s$ is an intermediate value between $q$ and $p$.

"In case of Gaussian criterion (type 6) the preference function remains increasing for all deviations and has no discontinuities, neither in its shape, nor in its derivatives. A parameter $\mathrm{s}$ has to be selected, it defines the inflection point of the preference function. We then recommend to determine first $a q$ and $a p$ and to fix s in between." (Brans and Mareschal, 2005)

The second segment: Determination of the estimated graph of higher rank

First, for each pair of solutions $\mathbf{x}^{1}, \mathbf{x}^{2} \in \mathbf{S}$, for each criteria function, the preference index of $x^{1}$ in relation to $\mathrm{x}^{2}$ is defined, assuming that each criterion is identified as one of the above criteria types. Thus the preference functions $P_{k}\left(\mathbf{x}^{1}, \mathbf{x}^{2}\right)$ are defined for each $k=1, \cdots, K$ (Kis the number of the criteria functions). In the case of determining the same importance for all criterion functions, the index of preferences is determined as: $\pi\left(\mathbf{x}^{1}, \mathbf{x}^{2}\right)=(1 / K) \sum_{k=1}^{K} P_{k}\left(\mathbf{x}^{1}, \mathbf{x}^{2}\right)$. This method allows decision makers to ${ }^{k=1}$ use weight coefficients with criteria functions: $w_{1}, w_{2}, \cdots, w_{K}$. Then it is

$$
\pi\left(\mathbf{x}^{1}, \mathbf{x}^{2}\right)=\left(\sum_{k=1}^{K} w_{k} P_{k}\left(\mathbf{x}^{1}, \mathbf{x}^{2}\right)\right) / \sum_{k=1}^{K} w_{k} .
$$

The preference indeks $\pi$ gives the measure of preference $\mathbf{x}$ over $\mathbf{x}^{2}$ for all criteria functions, and the closer to 1 it is, the higher the preference is.

Graph, whose core solutions are from S, such that for each $\mathbf{x}^{1}, \mathbf{x}^{2} \in \mathbf{S}, \operatorname{arc}\left(\mathbf{x}^{1}, \mathbf{x}^{2}\right)$, has the value $\pi\left(\mathbf{x}^{1}, \mathbf{x}^{2}\right)$, is called an estimated, ,higher rank“ graph.

\section{The third segment: use the relation of "higher} rank"

By using a higher ranking relation, the ranking of the solution in the partial order (PROMETHEE I) or in full order (PROMETHEE II) is performed. Here we will show how the ranking is done by the PROMETHEE II method.
For each solution $\mathbf{x}^{l} \in \mathbf{S}$ a net flow $\Psi\left(\mathbf{x}^{l}\right)$ is observed. $\Psi\left(\mathbf{x}^{l}\right)$ is calculated as

$$
\Psi\left(\mathbf{x}^{l}\right)=\Psi^{+}\left(\mathbf{x}^{l}\right)-\Psi^{-}\left(\mathbf{x}^{l}\right),
$$

that can simply be applied in the solutions ranking: $\mathbf{x}^{l}$ has a higher rang then $\mathbf{x}^{i}\left(\mathbf{x}^{l} P^{2} \mathbf{x}^{i}\right)$ if $\Psi\left(\mathbf{x}^{l}\right)>\Psi\left(\mathbf{x}^{i}\right)$ $\mathbf{x}^{l}$ is indifferent to $\mathbf{x}^{i}\left(\mathbf{x}^{l} I^{2} \mathbf{x}^{i}\right)$ if $\Psi\left(\mathbf{x}^{l}\right)=\Psi\left(\mathbf{x}^{i}\right)$, where $\Psi^{+}\left(\mathbf{x}^{l}\right)$ is the output flow obtained by:

$$
\Psi^{+}\left(\mathbf{x}^{l}\right)=\sum \pi\left(\mathbf{x}^{l}, \mathbf{x}^{i}\right), \quad i=1, \cdots, l,
$$

and $\Psi^{-}\left(\mathbf{x}^{l}\right)$ is the input flow obtained by:

$\Psi^{-}\left(\mathbf{x}^{l}\right)=\sum \pi\left(\mathbf{x}^{i}, \mathbf{x}^{l}\right), \quad i=1, \cdots, l$.

The PROMETHEE II method is a complete relation where all solutions are fully ranked. In addition to the methods of PROMETHEE I and II, the same authors have developed the additions, which are called PROMETHEE III, IV and V.

\section{APPLICATION OF MCPM INPRODUCTION PLAN OPTIMIZATION PROBLEM SOLVING}

\section{Multi-criteria programming problem}

The company Alfe-MI d.o.o. Živinice, $\mathrm{BiH}$ produces office and housing containers. The construction of the container is made of steel. Over $90 \%$ of production is contracted for the following year in advance. The data for forming multi criteria programming model are taken from Mandić (2016). We will here present the possibility and efficiency of using some multi criteria programming methods in the production plan optimization problem solving to help the decision maker to achieve the best level of his goals. The formed model will be solved using STEM and PROMETHEE methods.

\section{Multi-criteria programming model}

Let $\mathrm{x}_{\mathrm{j}}=$ the quantity of the $\mathrm{j}^{\text {th }}$ container in the production program $(j=1,2, \ldots, 7), f_{1}(\mathbf{x})=$ the total number of containers in the production program, and $\mathrm{f}_{2}(\mathbf{x})=$ the total gross profit for one year period. The multi criteria programming model is shown as

$$
\max _{\mathbf{x} \in \mathbf{S}} \mathbf{f}(\mathbf{x})=\left(\begin{array}{c}
f_{1}(\mathbf{x})=\sum_{j=1}^{7} x_{i} ; f_{2}(\mathbf{x})= \\
+262 x_{1}+1287 x_{2}+677 x_{3}+1526 x_{4}+3802 x_{5}+4352 x_{7} \\
+2901
\end{array}\right)
$$

where

$$
\mathbf{S}=\left\{\begin{array}{l}
\mathbf{x}=\left(x_{1}, \ldots, x_{7}\right)^{T}: 36 x_{1}+38 x_{2}+35 x_{3}+35 x_{4}+35 x_{5}+42 x_{6}+35 x_{7} \leq 257000 ; 8 x_{1}+8.5 x_{2}+ \\
+7 x_{3}+6.5 x_{4}+6.5 x_{5}+9 x_{6}+6.5 x_{7} \leq 56000 ; 8 x_{1}+8.5 x_{2}+8 x_{3}+8 x_{4}+8 x_{5}+8.5 x_{6}+8 x_{7} \leq \\
\leq 56000 ; 8.5 x_{1}+7.5 x_{2}+7 x_{3}+9 x_{4}+9 x_{5}+9 x_{6}+8 x_{7} \leq 56000 ; x_{1}+x_{2}+x_{3}+x_{4}+x_{5}+x_{6}+ \\
+x_{7} \geq 4650 ; x_{1}+x_{2}+x_{3} \geq 3850 ; x_{4}+x_{5}+x_{6} \geq 710 ; x_{7} \leq 100 ; 40 x_{1}-58 x_{2}=0 ; 2 x_{1}- \\
-58 x_{3}=0 ; 5 x_{4}-40 x_{5}=0 ; 55 x_{4}-40 x_{6}=0 ; x_{1}, \ldots, x_{7} \geq 0 \text { and integer }
\end{array}\right\}
$$


Multi-criteria programming model solving

We first solved the model as a linear integer programming problem, by using Excel Solver software to maximize each of the criteria functions individually on a given set of feasible solutions.

The following solutions are obtained:

Tabele 2 Optimal (marginal) solutions

\begin{tabular}{llcc}
\hline Solution & \multicolumn{1}{c}{ Variable values } & \multicolumn{2}{c}{ Ceiteria function values } \\
& \multicolumn{1}{c}{$f_{1}$} & $f_{2}$ \\
\hline $\mathbf{x}^{\mathbf{1}}$ & $x_{1}=3074, x_{2}=2120, x_{3}=106, x_{4}=288$ & 6079 & 8660865 \\
& $x_{5}=36, x_{6}=396, x_{7}=59$ & & \\
$\mathbf{x}^{\mathbf{2}}$ & $x_{1}=2291, x_{2}=1580, x_{3}=79, x_{4}=784$ & 6010 & 10108543 \\
& $x_{5}=98, x_{6}=1078, x_{7}=100$ & & \\
\hline
\end{tabular}

Source: Mandić (2016)

Table 1 shows that by maximizing the function $\mathrm{f}_{1}$, the maximum value obtained for this function differs significantly from the value obtained when the function $\mathrm{f}_{2}$ is maximized. Even greater difference in the criteria function values exists when the other criteria function $\mathrm{f}_{2}$ is maximized. This results indicate a lack of linear integer programming application in the optimization of the production program, as well as the necessity to apply a multi criteria programming method. By solving the presented model with the multi criteria programming methods, a compromise solution will be obtained that will give the acceptable values of the criteria functions for the decision maker.

\section{Solving the model by STEM method}

According the algorithm presented in the section 2.2., first we solved the following model

$\min _{(\mathbf{x}, \lambda) \in \mathbf{S}_{1}} \lambda$

using the calculated

$\alpha_{1}=0.00429, \alpha_{2}=0.0000207248, \pi_{1}=0.995$ and

$\pi_{2}=0.005$, where

$$
\mathbf{S}_{1}=\left\{(\mathbf{x}, \lambda) \in \mathbf{R}^{n+1}: \mathbf{x} \in \mathbf{S} ; \lambda \geq\left|6079-f_{1}(\mathbf{x})\right| \cdot 0.995 ; \lambda \geq\left|10108543-f_{2}(\mathbf{x})\right| \cdot 0.005 ; \lambda \geq 0\right\}
$$

The following solution has been obtained: $\mathrm{f}_{1}=6010$, $\mathrm{f}_{2}=10108543$.

The DM was not satisfied with the obtained valuefor the criteria function $\mathrm{f}_{1}$.
In the second step of the STEM method we used the following inputs: $\pi_{1}=1, \pi_{2}=0$

$$
\Delta f_{2}^{c}=\left\{\begin{array}{l}
208543 ; 308543 ; 408543 ; 508543 ; 608543 ; 708543 ; 808543 ; \\
908543 ; 1008543 ; 1108543 ; 1288543 ; c=1, \ldots, 11
\end{array}\right\} . \text { Therefore the following }
$$

models are solved:

$$
\min _{(\mathbf{x}, \lambda) \in \mathbf{S}_{2}^{c}} \lambda
$$

where

$$
\mathbf{S}_{2}^{c}=\left\{(\mathbf{x}, \lambda) \in \mathbf{R}^{n+1}: \mathbf{x} \in \mathbf{S} ; \lambda \geq 6079-f_{1}(\mathbf{x}) ; f_{2}(\mathbf{x}) \geq 10108543-\Delta f_{2}^{c} ; f_{1}(\mathbf{x}) \geq 6010 ; \lambda \geq 0\right\}
$$


The obtained results are presented in Table 3.

Table 3 Results after step 2 of the STEM method

\begin{tabular}{cccc}
\hline Solution & $\Delta f_{2}^{c}$ & $f_{1}$ & $f_{2}^{\text {nd }}$ \\
\hline I & 208543 & 6019 & 9957800 \\
II & 308543 & 6027 & 9802716 \\
III & 408543 & 6030 & 9702164 \\
IV & 508543 & 6035 & 9647632 \\
V & 608543 & 6040 & 9555762 \\
VI & 708543 & 6049 & 9405019 \\
VII & 808543 & 6050 & 9333123 \\
VIII & 908543 & 6056 & 9245594 \\
IX & 1008543 & 6060 & 9110484 \\
X & 1108543 & 6070 & 9002981 \\
XI & 1208543 & 6072 & 8935426 \\
\hline
\end{tabular}

Source: Authors' calculations

From the set of non-dominated solutions presented in Table 2, the decision maker should choose the preferred solution. However, if the decision maker is not sure about which solution is the best for him, he can give some information to the analyst who will apply the PROMETHEE method and help the decision maker to choose the preferred solution.

\section{Solving the model by PROMETHE method}

Table 3 presents the set of 11 non-dominated solutions. To help the decision maker to choose the best (preferring) solution from the given set we used the Decision Lab Software. The decision maker chose type 6 of criteria for both criteria functions with $\mathrm{s}=3$ and the weight coefficient $\mathrm{w}_{1}=\mathrm{w}_{2}=1$.

The obtained results are given in the figure 1 .

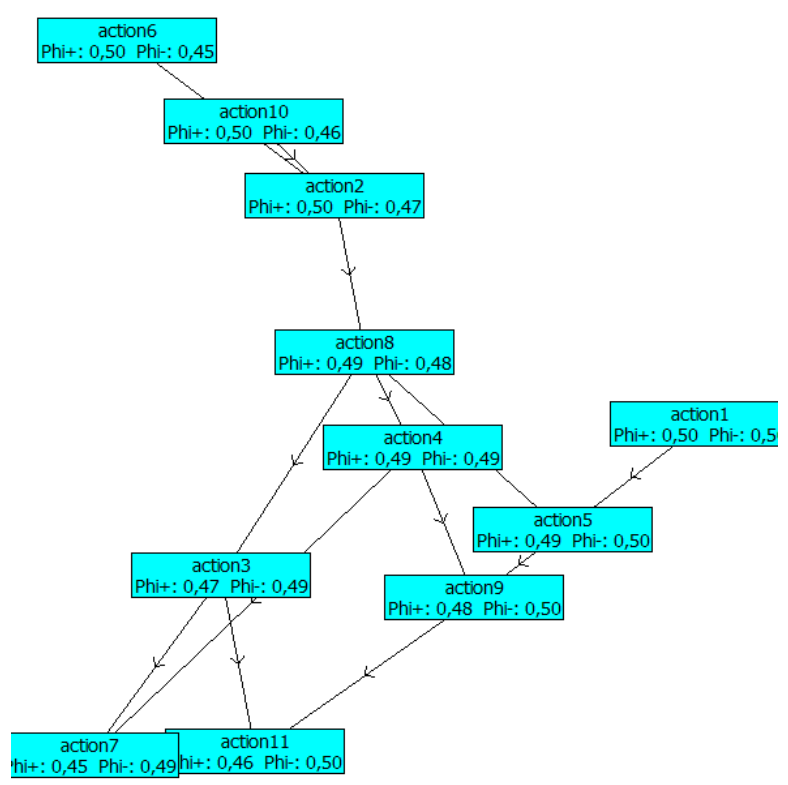

Figure 1. PROMETHEE network 
From the Figure 1 we can see that the solution (action) $\mathbf{x}^{6}$ has the biggest net flow (0.03) and the solutions $\mathbf{x}^{10}, \mathbf{x}^{2}$ and $\mathbf{x}^{8}$ have positive net flow. Therefore, the action 6 is the best for the decision maker.

The Decision Labsoftware allows us to analyze the sensitivity of the solutions obtained by changing the parameter $s$, changing the function type and their parameters $q$ and $p$ for the criteria functions and changing the coefficients $w_{1}$ and $w_{2}$. We conducted the analysis with regard to the value of the coefficients $w_{1}$ and $w_{2}$, and we concluded that regardless of the change in coefficient values $w_{1}$ and $w_{2}$, the solution $\mathbf{x}_{6}$ always has rank 1.

\section{CONCLUSIONS}

The application of STEM and PROMETHEE methods in solving the problem of optimization of the production plan in the metal industry indicates the possibility of their efficient application. We applied the STEM methodto create a set of non-dominated solutions, whilewe used the PROMETHEE method to select a preferred solution from a set of non-dominated solutions. The STEM method allows creating a large number of non-dominated solutions without the active participation of the decision maker. The PROMETHEE method allows the choice of the best (preferred) solution from a set of non-dominated solutions, whereby the decision maker is required to determine the type of criteria that would match the particular criteria function and the parameters of indifference and preference if the decision maker can determine it.

The PROMETHEE method allows the analysis of the sensitivity of the rank of the obtained solutions in relation to the changes in the weights of the criteria functions. In addition, the method analyzessensitivity of the changes of the function types related to the particular criteria function and the change of parameters of indifference and preference, which significantly helps the decision maker when choosing the preferred solution.

Based on the presented research we can conclude that the application of the STEM and PROMETHEE methods has a high application efficiency of the production plan optimization of the company from the metal industry.

The investigation of application efficiency of multicriteria programming methods in solving complex multi-criteria programming models with a big number of variables and constraints, we propose for the future research.

\section{REFERENCES}

Brans, J-P. \& Vincke, P. (1984). A Preference Ranking Organization Method, the PROMETHEE method. Management Science, 31, pp. 647-656.

Brans, J-P. \& Mareschal B. (2005). "PROMETHEE Methods", in Multiple Criteria Decision Analysis: State of the Art Survey (Edited by: Jose Figueira, Salvatore Greco and Matthias Ehrgott). New York: Springer.

Chankong, V., Haimes, Y. Y. (1983). Multiobjective Decision Making: Theory and methodology. New York: North Holland.

Hwang, C. L., \& Masud, A. S. M. (1979). Multiple Objective Decision Making : Methods and Applications. New York: Springer Verlag.

Lai, Y. J. \& Hwang, C. L. (1996). Fuzzy Multiple Objective Decision Making. Berlin: Springer.

Mandić, Ž. (2016). Production Optimization in Metal Industry by using Multi Criteria Programming Methods, Master's Thesis, University of Tuzla, Faculty of Economics, Tuzla. (in Bosnian)

Opricović, S. (1986). Multi Criteria Optimization. Beograd: Naučna knjiga. (in Serbian)

Perić, T. (2008). Multi Criteria Programming - Methods and Applications, Zagreb: Alka script.

Steuer, R. E. (1985). Multiple Criteria Optimization. New York: Willey.

Zionts, S. (1980). "Methods for Solving Management Problems involving Multiple Objectives", in Multiple Criteria Decision Making: Theory and Applications, Koenigswinter, West Germany, 1979 (Editors: G. Fandel and T. Gal). New York: Springer Verlag. pp. 540-558. 